\title{
Inclusion cleanliness in metallic alloys
}

\author{
Jean-Pierre Bellot ${ }^{1, *}$, Pascal Gardin ${ }^{2}$, and Frédéric Gruy ${ }^{3}$ \\ ${ }^{1}$ Université de Lorraine, CNRS, IJL, Laboratory of Excellence DAMAS, 54000 Nancy, France \\ 2 ArcelorMittal Global R\&D Maizières, BP30320, 57283 Maizières-les-Metz Cedex, France \\ 3 Mines Saint-Étienne, Univ Lyon, CNRS, UMR 5307 LGF, Centre SPIN, 42023 Saint-Étienne, France
}

Received: 9 May 2019 / Accepted: 9 May 2019

The inclusion cleanliness remains an issue of great concern in liquid metal processing which has been addressed in a full session of the last Matériaux Conference held in November 2018 in Strasbourg, France. A special issue of the Metallurgical Research E Technology journal gathers most of the papers of this session which are divided into two sub-sections: on the one hand the analysis and characterization techniques of the inclusions are addressed and on the other hand the modelling methods to simulate the behavior of single particle or a population of inclusions are discussed.

Among the recurring sources of concern of the metallurgical industry remain the characterization and control of the inclusion cleanliness. Most of the time, in-use properties of metals and alloys are affected by isolated or clustered inclusions. Then, it is not surprising that the industry of aluminum, steel, reactive metals and superalloys are equally concerned by the cleanliness issues. Whatever the situation, the size, the morphology and the chemical composition of the particles play a significant role. However, the requirements in terms of inclusion cleanliness are more and more difficult to achieve as the objectives of lightweighting of metallic parts, of mechanical performance and of cost reduction are pursued. The necessary steps to progress concerns both the detection techniques and inclusion characterization (often automated), and the breakthroughs in the simulation methods of inclusion behavior in liquid metal processing. Those developments will make easier the emergence of new solutions for the control of inclusion cleanliness at the industrial scale.

The Conference Matériaux2018 held November 19-23, in Strasbourg, France, gathered around 2000 attendees. Among the large variety of topics, a workshop on Inclusion cleanliness in metallic alloys was organized. From the papers presented at this workshop, 13 were selected and reviewed for publication in the special issue of the Journal of Metallurgical Research \& Technology to highlight the key scientific and engineering advances in the field.

This special issue is divided into two complementary topics, on the one hand the analysis and characterization techniques of the inclusions are addressed and on the other hand the modelling methods to simulate

\footnotetext{
* e-mail: jean-pierre.bellot@univ-lorraine.fr
}

the behavior of single particle or a population of inclusions.

\section{Part 1: Analysis and characterization}

These techniques cover the non-destructive detection methods such as ultrasonic testing and X-ray tomography, which have made significant progress in terms of detection performance and definition, as well as all the post-mortem methods using quantitative metallography which remain nowadays often employed.

The analysis automation requires high level of statistical treatments providing many internal properties of the inclusion population (composition, morphology...). The simulation of the measure is now a large area of investigation (3D prediction from 2D measurements, assessment of the measure uncertainty, theory of extreme values...) (Fig. 1).

\section{Part 2: Modelling of inclusion behavior in liquid metal processing}

The numerical simulation of dispersed phases is undergoing an important scientific development that benefits simulation of inclusions in the metallurgical reactors. New numerical techniques are in progress today addressing complex issues at different scales (from the mesoscopic scale of the inclusion to the macroscopic scale of the process), combining both thermodynamic and fluid dynamic aspects. Moreover, the interaction between inclusions (collision, aggregation, fragmentation) and the behavior of inclusions at interfaces (solidification front, bubble, slag...) remains significant scientific challenges. 


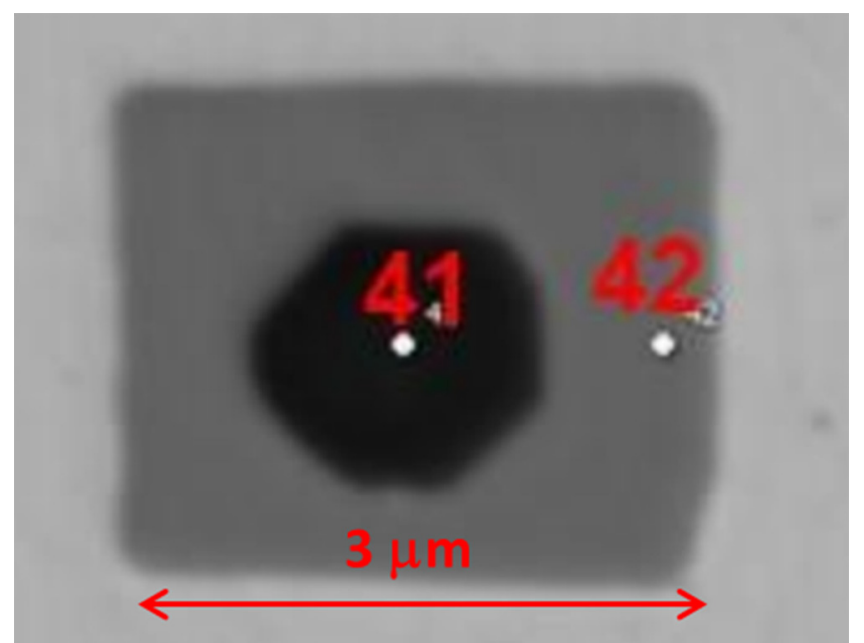

Fig. 1. SEM + EDS analysis of a TiN precipitate around a core of $\mathrm{Al}_{2} \mathrm{O}_{3}$ (E. Lucas et al., MR\&T, 2019).

Finally, the thermochemical equilibrium between the inclusions and their environment (liquid metal, slag or gas) are addressed by many numerical software and databases in continuous development. All these topics

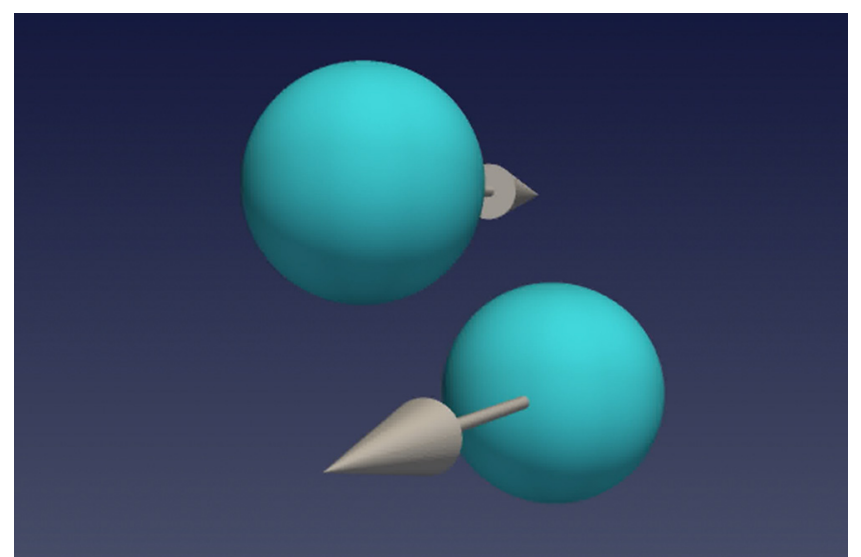

Fig. 2. Direct Numerical Simulation of possible crossing of an inclusion pair in a pure shear flow (Courteously from M. Gisselbrecht, IJL France).

include also the behavior of inclusions after solidification, with the growth of inclusions in grain boundaries and their evolution during the thermomechanical treatments as well (Fig. 2).

Cite this article as: Jean-Pierre Bellot, Pascal Gardin, Frédéric Gruy, Inclusion cleanliness in metallic alloys, Metall. Res. Technol. 116, 507 (2019) 